\section{Suzanne Maloney: The Roots and Evolution of Iran's Regional Strategy, Washington, D.C.: Atlantic Council, 2017.}

Besprochen von Remko Leemhuis, AJC Berlin Ramer Institute, Consultant on Antisemitism Affairs, Berlin, Germany;

E-mail: LeemhuisR@ajc.org

https://doi.org/10.1515/sirius-2018-2018

Die Hoffnungen, dass eine Einigung im Streit um das iranische Atomwaffenprogramm die Möglichkeit eröffnet, mit Teheran auch auf anderen Konfliktfeldern ins Gespräch $\mathrm{zu}$ kommen und bestenfalls diplomatische Lösungen $\mathrm{zu}$ finden, waren groß - insbesondere in Europa. Dabei versprachen sich die europäischen Diplomaten, kurz und mittelfristig die aggressive Politik des Mullah-Regimes im Nahen Osten einhegen zu können. Drei Jahre nach Abschluss des JCPOA ist von diesen Hoffnungen wenig geblieben, verfolgt das Regime seine hegemoniale Politik in der Region doch aggressiver denn je. Der Frage, welches die wesentlichen Determinanten sind, die diese iranische Außenpolitik beeinflussen geht Suzanne Maloney in dem vorliegenden Papier nach. Dabei identifiziert sie fünf wesentliche Elemente: die imperiale Geschichte des Iran, den schiitischen Islam, Antiimperialismus, innenpolitische Aspekte sowie die Bestrebungen des Regimes, sein Überleben zu sichern.

Der Autorin ist zuzustimmen, wenn die darauf hinweist, dass der Iran den Nahen Osten unter so gut wie jeder Regierung als sein natürliches Einflussgebiet sehen und sich als Gestaltungsmacht verstehen würde. Dieses Sendungsbewusstsein speist sich aus der Geschichte der persischen Reiche. Der zuweilen chauvinistische Stolz auf die vergangene zivilisatorische und imperiale Bedeutung ist bis heute tief im Selbstverständnis des Regimes wie auch weiter Teile der Bevölkerung verankert. Gleichsam beeinflusst es das strategische Denken des Regimes, insbesondere wenn es um seine Nachbarstaaten geht. So äußerte ein ehemaliger Minister: "[Iraq is] not only part of our civilizational influence, but it is our identity, culture, center and capital... Because Iran and Iraq's geography and culture are inseparable.”

Die Selbstwahrnehmung als hegemonialer Akteur im Nahen Osten wurde nach der Islamischen Revolution 1979 noch durch ein schiitisch-religiöses Sendungsbewusstsein ergänzt und verstärkt. Als Motor der Revolution und Grundlage des gesamten politischen Systems identifiziert Maloney die revolutionäre Theologie Khomeinis. Stand und steht der überwiegende Teil des schiitischen Klerus politischer Macht und Einflussnahme stets skeptisch bis ablehnend gegenüber, betrachtete Khomeini die Revolution im Iran nur als den Anfang einer Umwälzung des gesamten Nahen Osten. Nach dem Vorbild des Iran sollten alle „korrupten“, „westlichen“ und „ungerechten“ Regime der Region gestürzt und durch politische Systeme nach dem Vorbild des post-revolutionären Iran ersetzt werden. Auch wenn es nicht gelungen ist, dieses politische System in einem anderen Land $\mathrm{zu}$ etablieren, konnte Teheran zweifellos seinen Einfluss auf die schiitischen Minderheiten in den mehrheitlich sunnitischen Staaten ausweiten. Gleichzeitig hat das Regime damit die konfessionellen Spannungen zwischen Schiiten und Sunniten verstärkt. Treffend weist Malony darauf hin, dass die Mobilisierung weit weitverbreiteter antisemitischer Ressentiments und die Selbstpräsentation als Vorkämpfer aller Muslime gegen Israel auch zur Abkühlung dieser Spannungen beitragen sollen. Jedoch greift diese Analyse allerdings insofern zu kurz, als der Antisemitismus eben nicht nur ein Instrument Teherans ist, sondern tief in der schiitischislamistischen Ideologie verankert ist und die Weltsicht der Herrschenden in Teheran wesentlich prägt.

Im Zusammenhang mit der Feindschaft gegen den jüdischen Staat porträtiert sich das Regime ebenso stets als Bollwerk gegen den Imperialismus, das den Nahen Osten vor westlicher Ausbeutung schützt. Auch wenn der Iran nie eine Kolonie war, gab es immer wieder Versuche von außen, auf die inneriranischen Angelegenheiten einzuwirken. Diese historische Erfahrung ist ein zentraler Grund für die Vehemenz, mit der die Mullahs den Iran von tatsächlicher und vermeintlicher äußerer Einflussnahme schützen. Als Hauptfeinde der Islamischen Republik werden dabei, auch nicht ganz zu Unrecht, vor allem die USA als „großer Satan“ und Israel als „kleiner Satan“ ausgemacht. Dass diese Feindschaft seit 1979 nahezu ausschließlich von Teheran befeuert wird, erwähnt Maloney indes nicht. Zurecht weist sie aber auf die Ironie hin, dass das Regime zwar vehement seine staatliche Souveränität verteidigt, in der Region selber aber als imperiale Macht agiert und die Souveränität anderer Länder, wie etwa im Irak, Syrien, Jemen und dem Libanon deutlich wird, nicht respektiert.

Aber auch die Innenpolitik spielt eine maßgebliche Rolle bei der Formulierung und Gestaltung der Außenpolitik. Maloney stellt fest, dass die Ursache für dieses Übergreifen in der Konkurrenz der verschiedenen Frak- 
tionen innerhalb des politischen Systems liegt. Dieses polykratische System, so schreibt sie, war lange Zeit ein Schwachpunkt - so etwa und vor allem während des IranIrak-Krieges, als das unübersichtliche System staatlicher und außerstaatlicher Institutionen die militärische Verteidigungsfähigkeit erheblich beeinträchtigte. Mittlerweile hat sich diese Konkurrenz im System indes als ein Vorteil herausgestellt, kann der Iran über die Revolutionsgarden und die Quds-Einheiten doch langanhaltend und mehrdimensional in anderen Ländern agieren. Hier sei vor allem an den Libanon erinnert, wo der Iran über die Hisbollah nicht nur politisch einen erheblichen Einfluss ausübt, sondern mit dieser auch über eine beachtliche militärische Schlagkraft verfügt.

Als letzten und wichtigsten Aspekt sieht Maloney den Willen der Herrschenden, das Überleben des Regimes zu sichern, womit eine gewisse Paranoia verbunden ist. Die historische Erfahrung erfolgter oder zumindest versuchter Lenkung Irans durch andere Staaten leistet dabei einem verschwörungsideologischen Blick auf die Welt Vorschub, der den Motiven anderer fortwährend misstraut und stets eine verborgene Agenda vermutet, die sich letztlich und im Kern gegen die Existenz der Islamischen Republik richtet. Diese paranoide Wahrnehmung der Umwelt ist auch einer der Gründe dafür, dass das Regime etwa in Syrien erhebliche Ressourcen aufwendet, sehen doch die Mullahs in dem Aufstand gegen das Assad-Regime schließlich den Versuch rivalisierender Akteure, namentlich Israels und der USA, einen Verbündeten zu stürzen und den Iran zu schwächen - mit dem langfristigen Ziel, dort ebenfalls einen Regime Change herbeizuführen.

Maloneys Studie hebt sich wohltuend von Analysen aus Deutschland und Europa ab, da sie die Rolle der Ideologie deutlich hervorhebt. Gerade in der hiesigen Debatte werden diese Aspekte stets heruntergespielt oder als Beiwerk betrachtet, hinter dem sich realpolitische Ziele verbergen. Maloney zeigt hingegen schlüssig, dass etwa der Hass auf den Westen, der Antisemitismus und die revolutionäre-islamistische Ideologie ernstzunehmende Faktoren sind, die im Umgang mit dem Iran berücksichtigt werden müssen.

http://www.atlanticcouncil.org/publications/issuebriefs/the-roots-drivers-and-evolution-of-iran-s-regionalstrategy 